\title{
Platooning of Autonomous Vehicles with Intervehicle Communications in SUMO Traffic Simulator
}

\author{
Pedro Fernandes Student Member, IEEE, and Urbano Nunes, Senior Member, IEEE
}

\begin{abstract}
An approach to increase road capacity consists in making vehicles move much closer to each other, i.e. using platoons, and to avoid the constant stop-and-go in urban traffic. This paper addresses the studies of new models to allow the research of cooperative and autonomous communication-enabled vehicles, with platooning capabilities, and the addition of new features in SUMO (Simulation for Urban MObility) to allow the simulation of those models. Simulations results of vehicle platoons using constant spacing instead of the more common constant time headway, with intervehicle communications, are reported in the paper. The platoon leaders are controlled by an external application and the simulation platform works at the subsecond level.
\end{abstract}

\section{INTRODUCTION}

Intervehicle communications (IVC), namely vehicle-tovehicle communications (V2V), allow vehicles to exchange data among them, enabling a more efficient road network use [1]. However, in urban areas, congestion is a major issue and expansion of the road network is frequently not a viable option anymore. Even Advanced Traffic Information Systems (ATIS), using GPS data and transmission of better route options through IVC may be of limited use, due to the overcapacity that urban road networks are facing nowadays. One viable approach to increase road capacity consists of making vehicles move much closer to each other, i.e. using platoons. Additionally, eliminating the constant stop-andgo of actual traffic, whether on intersections or at transit stops, would avoid wasting time and energy consumption. To be really effective, such system should be based on driverless vehicles. The major reason for such procedure is related with human factors such as the reaction time, diverse behavior among drivers (some careful, others driving aggressively) which can cause traffic disturbances, and limited attention to nearby traffic (worsened by the increasing use of smartphones and PDA's while driving). Such a system would benefit considerably with the use of IVC-enabled autonomous vehicles, which could enable a very low reaction time and tight coordination among them.

Traffic simulations already gave a major contribution to the study and forecast of traffic behavior. Most traffic simulators model human driving behavior, e.g. car following and Intelligent Driving Model (IDM) [2] and, more recently, the Human Driving Model (HDM) [3], to evaluate traffic

This work was supported by Portuguese Foundation for Science and Technology under Grant PTDC/SEN-TRA/099413/2008. The work of P. Fernandes was supported by Portuguese Foundation for Science and Technology through the research fellowship SSFRH/BD/38605/2007.

The authors are with the Institute of Systems and Robotics, Department of Electrical and Computer Engineering, University of Coimbra, 3030-290 Coimbra, Portugal (e-mail: pedro@isr.uc.pt; urbano@isr.uc.pt). conditions in foreseeable scenarios. However, simulation of autonomous vehicles with IVC is an important field where new research scenarios may take place.

Direct Short Range Communications (DSRC/WAVE/IEEE 802.11p) [4] present some features that are appropriate to $\mathrm{V} 2 \mathrm{~V}$, namely its low latency and reasonable range. However, multihop broadcast schemes in DSRC are still a subject of vast research, particularly when the message receivers are beyond the effective DSRC transmission range, leading to the need of vehicles to relay broadcasted messages [5], [6]. Nevertheless, DSRC is suitable in short range communications, e.g. in safety or vehicle coordination related tasks.

A transport system based on autonomous vehicles coordination, and routing and congestion control of the road network. These and other requirements demand a communication channel between vehicles and the infrastructure. To accomplish these purposes, DSRC, although encompassing vehicle-to-infrastructure (V2I) features, might be usefully complemented by the use of Long Term Evolution (LTE) and LTE-Advanced communications [7], [8], [9].

This research encompasses the expansion of SUMO [10] features, to allow studies on cooperative behaviors of communication-enabled autonomous vehicles. Moreover, using the new SUMO capabilities, the study of autonomous vehicles platoons with constant spacing [11] with intervehicle communications, has been addressed.

In the present stage of this research, some assumptions are made. Firstly, only longitudinal control of vehicles is assumed. This is in line with autonomous vehicles using dedicated tracks, whether on ground or elevated, as is the case of Personal Rapid Transit (PRT) systems [12]. Secondly, since the platoon dimension is about $30 \mathrm{~m}$ long (8 vehicles of $3 m$ with $1 m$ apart) and DSRC lower range is usually well above that value, we assume flawless communications due to transmission range. Finally, as our communication algorithm uses time slots coordinated by the platoon leader, no packet collisions are envisaged, at least those related with intraplatoon communications. For that reason, we find no need for the use, at the present stage, of a network simulator, such as the NS2 [13].

PRT systems require further comments. These systems encompass many interesting possibilities as shown in Table I (some of the items from [12]). Its implementation, experimental testing and field applications have been described [14]. However, the net benefits of this mode of transit with regard to light rail or cars are yet to be clearly demonstrated.

Nevertheless, the recent technological advances, namely on autonomous vehicle control, EVs, communications 
TABLE I

PERSONAL RAPID TRANSIT MAIN CHARACTERISTICS

\begin{tabular}{l}
\hline \hline Characteristics \\
\hline Off-line stations \\
Minimum-sized and minimum weight vehicles \\
Adequate speed to maximize network capacity \\
Fully automatic control \\
Hierarchical, modular, asynchronous control \\
Dual-redundant communication technologies (V2V and V2I) \\
Accurate position and speed sensors \\
Vehicle movement when requested by the users \\
Automatic routing of empty vehicles to manage unbalanced demand \\
Nonstop trips from origin to destination \\
Guideways and electrical propulsion \\
High level of privacy and safety
\end{tabular}

(DSRC, LTE), are introducing new elements to the equation of whether its advantages or disadvantages are enough to consider PRT systems as an option for sustainable urban mobility. Moreover, such systems may present other favorable characteristics if relying on autonomous EVs. In fact, since the vehicles may evolve very close to each other, forming platoons, a considerable reduction on energy consumption might be expected [12], because each follower vehicle would benefit from a reduced air drag due to their precedent vehicle (and, in much lower extent, even the leader's air drag might be slightly reduced, if its rear turbulence is reduced as well). Furthermore, platooning enables a considerable increase of lane capacity. Moreover, if conceived as an elevated system with off-line stations, PRT eliminates the stop-and-go problem of common car traffic and transit systems, contributing to a faster and more comfortable mobility with higher energy efficiency. Last, but not the least, as the proportion of elderly is growing in the western countries, urban mobility claims for a new paradigm, for which these systems may contribute positively. An urban transportation system based on autonomous vehicles, such as a pool of Cybercars [15] or PRT, could enable inhabitants to regain the city space.

Our main contribution consists in the study of new models to allow the research of cooperative and autonomous communication-enabled vehicles, with platooning capabilities. Moreover, to enable significant increase of road capacity, the constant-spacing platooning method with intervehicle communications is studied and used. The improvement of the SUMO traffic simulator with the implementation of the aforementioned models is also performed.

\section{RELATED WORK}

This research encompasses several fields of knowledge (as shown in Table II). Pravin Varaiya, in [19], discussed key issues related to an highly automated IVHS (Intelligent Vehicle/Highway System) and proposed a IVHS control system architecture. Swaroop [20] investigated various platooning control strategies and their impact on the performance of platooning. Alvarez and Horowitz [21] researched the conditions to achieve safe platooning under normal mode
TABLE II

FIELDS OF KNOWLEDGE TO CONSIDER

\begin{tabular}{ll}
\hline \hline Field & Some relevant topics \\
\hline Traffic & Theory, fundamental diagram, flow, capacity \\
Communications & DSRC/WAVE/IEEE 802.11p, LTE (4G) \\
Simulation & Traffic, comm. networks, multi-agent based \\
Control & Constant spacing, string stability \\
Autonomous vehicles & Communicant, cooperative \\
Computer Science & C++, Python, Java, application architecture \\
Energy & Consumption, efficiency \\
\hline
\end{tabular}

of operation. Horowitz and Varaiya describes in [22] a fivelayer AHS control system architecture, involving the infrastructure and the vehicles. Rajamani et al. [23] developed a controller of platoons with constant spacing, concluding that autonomous control is not enough to ensure string stability. $\mathrm{He}$ found that only with IVC is the string stability of vehicle platoons achieved. The method was experimentally evaluated in field tests.

The effects of communication delays on string stability of platoons was researched by Mahal [25]. The main conclusion was that string stability of platoons is compromised in the presence of communication delays. A simple method to deal with string instability due to communication delays is proposed in [26].

Traffic simulation has been a major field of research and development. MITSIM, MATSIM, SUMO, TRANSIMS, PARAMICS, AIMSUN2, ARTEMiS, INTRAS, CORSIM, CARSIM, INTELSIM, VISSIM and CARISMA, are a few of the simulators that have been used to perform research on traffic analysis and control. Some of them are (or have versions of) open source, such as MITSIM, MATSIM, SUMO and TRANSIMS. The MATSIM [27], [28] is also developed under the multi-agent paradigm, which is of great relevance, if the emergence of traffic behavior under certain traffic demands is the subject of research. SUMO [10], [29], [30] is an open source traffic simulator which is based on the Gipps-model extension [31] and, more recently, also on the IDM model. Most of the aforementioned simulators have already reached a mature state of development. The paradigm under their development and architecture relies mainly on human driving behavior, and the traffic models are based on conventional cars and buses evolving on classical roads with intersections, semaphores and increasingly traffic congestion. IVC is a very promising technology that may improve traffic flow and reduce congestion. Research on the use of IVC and its implications on traffic routing have been pursued. For example, a system which integrates a traffic simulator (CARISMA), the network simulator NS2 [13] and intervehicle messages exchanging is described in [32]. Piorkowski et al. proposed TraNS [33], a vehicular network simulator that integrates the SUMO and the NS2 network simulator. In the same line of research, Wegener et al. proposed TraCI [34], coupling the SUMO with other applications through a TCP-based client-server architecture. 
Recently, simulators more focused on PRT systems have been proposed [35], [36].

However, new traffic simulator approaches are required for enabling accurate research addressing issues such as: formation of wireless IVC-enabled autonomous vehicle platoons with constant spacing; coordination of platoons; vehicle's energy consumption studies; comparison studies between IVC-enabled autonomous vehicle platoons with analogous traffic demands served by conventional traffic (whether of private or public nature).

\section{BASE MODEL OF VEHICLE CONTROL}

Maintaining a desired intervehicle spacing in platoons demands a tight control of each vehicle. In case of humandriven vehicles, intervehicle spacing is defined through a constant time headway, which means that increasing speed implies increased spacing. According to [3], the 'desired minimum gap' between two vehicles under IDM, is given by

$$
s^{*}(v, \Delta v)=s_{0}+v T+\frac{v \Delta v}{2 \sqrt{a b}}
$$

where $\alpha$ denotes the following vehicle, $\alpha-1$ denotes the leading vehicle, $\Delta v:=v_{\alpha}-v_{\alpha-1}, s_{0}$ is the minimum distance for congested traffic, $a$ the maximum acceleration and $b$ the 'comfortable deceleration'. The last term of (1) is only significant in non-stationary traffic, when $\Delta v \neq 0$. The term $v T$ is the more relevant one to the resultant spacing in stationary traffic, where $T$ denotes the 'safety time gap'. When speed is higher, $s^{*}$ increases proportionally, which means that the road vehicle density decreases. With human drivers, it is not possible to avoid this performance pattern, even with IVC, because human reaction time is the main reason for the existence of the safety gap proportional to the vehicle speed. The human reaction time, typically in the order of magnitude of one second, leads to the lack of string stability in vehicle platooning. This means that, if the lead vehicle decelerates, the spacing between vehicles may decrease toward the end of the platoon, leading to possible collisions.

According to [19], road capacity may be increased by the use of tightly spaced intra-platoon vehicles. His formulation to determine road capacity is as follows:

$$
C=v \frac{n}{n s+(n-1) d+D}
$$

where $d$ represents the intra-platoon spacing, $D$ the interplatoon spacing, $s$ the vehicle length, $v$ the steady-state speed, and $n$ the number of cars in each platoon.

Based on (2), Table III presents some values, with $s=3 \mathrm{~m}$ and velocities of $36 \mathrm{~km} / \mathrm{h}$ and $72 \mathrm{~km} / \mathrm{h}$. The spacing of free vehicles $(n=1)$ is consistent with the minimum gap from the time headway model, in which human drivers, presenting reaction times between $0.25 s$ and $1.2 s$ seconds, need, at least $12 \mathrm{~m}$ of safety gap at a speed of $36 \mathrm{~km} / \mathrm{h}$. Note that even for an inter-platoon spacing double of the spacing of individual vehicles, the improvement of road capacity with, for instance, platoons of 8 vehicles each is more than doubled, compared
TABLE III

\begin{tabular}{|c|c|c|c|c|c|}
\hline $\mathbf{v}$ & $\mathbf{n}$ & 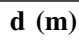 & D (m) & C (ve/h) & Improv. \\
\hline \multirow{6}{*}{$\frac{\hat{D}}{0}$} & 1 & - & 15 & 2000 & - \\
\hline & 5 & 1 & 30 & 3674 & 1.8 \\
\hline & 8 & 1 & 30 & 4721 & 2.4 \\
\hline & 15 & 1 & 30 & 6067 & 3.0 \\
\hline & 20 & 1 & 30 & 6606 & 3.3 \\
\hline & 100 & 1 & 30 & 8392 & 4.2 \\
\hline \multirow{6}{*}{$\frac{\sum}{N}$} & 1 & - & 30 & 2182 & - \\
\hline & 5 & 1 & 30 & 7347 & 3.4 \\
\hline & 8 & 1 & 30 & 9443 & 4.3 \\
\hline & 15 & 1 & 30 & 12135 & 5.6 \\
\hline & 20 & 1 & 30 & 13211 & 6.1 \\
\hline & 100 & 1 & 30 & 16783 & 7.7 \\
\hline
\end{tabular}

RoAD CAPACITY USING Platoons

TABLE IV

Nomenclature of Mathematical Formulations

\begin{tabular}{ll}
\hline \hline Symbol & Description \\
\hline$\ddot{x}_{i d e s}$ & desired acceleration of the $i$ th vehicle \\
$\ddot{x}_{i-1}$ & acceleration of the vehicle preceding the $i$ th vehicle \\
$\ddot{x}_{l}$ & acceleration of the vehicle leader of the platoon \\
$V_{i}$ & velocity of the $i$ th vehicle \\
$V_{l}$ & velocity of the vehicle leader of the platoon \\
$C_{1}$ & control gain used in longitudinal controller \\
& (weight factor of the leader with respect to the \\
$\xi$ & preceding vehicle, with values $0<C_{1}<1$ ) \\
$\omega_{n}$ & damping ratio (typically equal to 1 for critical damping) \\
$S_{i}$ & bandwidth of the controller \\
\hline
\end{tabular}

with the free vehicle case. When doubling the velocity in Table III, the capacity improvement is residual (9\%) in the case of free vehicles. However, the capacity almost doubles with platoons of 8 vehicles ( $83 \%$ better), when compared with the analogous case at the speed of $36 \mathrm{~km} / \mathrm{h}$, resulting in more than four times the capacity of the road with free vehicles.

It becomes clear that one way to effectively improve road capacity consists on the use in vehicle platoons with constant spacing. However, to ensure string stability between vehicles in a platoon, IVC is required, since string stability can not be ensured by autonomous control [11].

Considering the desired space between vehicles defined by

$$
x_{i_{-} d e s}=x_{i-1}-L_{i}
$$

with $L_{i}$ being a constant that includes the length of the preceding vehicle $l_{i-1}$, Rajamani [11] defines the spacing error as:

$$
\varepsilon_{i}=x_{i}-x_{i-1}+L_{i}
$$

Using a sliding mode approach, Rajamani designed a controller [11] with the desired acceleration of vehicles expressed as: 


$$
\begin{aligned}
\ddot{x}_{i \_d e s}= & \left(1-C_{1}\right) \ddot{x}_{i-1}+C_{1} \ddot{x}_{l} \\
& -\left(2 \xi-C_{1}\left(\xi+\sqrt{\xi^{2}-1}\right)\right) \omega_{n} \dot{\varepsilon}_{i} \\
& -\left(\xi+\sqrt{\xi^{2}-1}\right) \omega_{n} C_{1}\left(V_{i}-V_{l}\right)-\omega_{n}^{2} \varepsilon_{i}
\end{aligned}
$$

The parameters of (5) are described in Table IV. With this control method, spacing error will converge to zero [11]. From (5) we can conclude that, to determine the desired acceleration of each vehicle, wireless communications are needed, to allow each vehicle to receive the speed and acceleration values from both the preceding vehicle and the platoon leader.

\section{SIMULATOR}

For our multi-vehicles studies in urban scenarios, the open source SUMO traffic simulator was selected [10]. SUMO can be connected with other applications through a TCPbased client-server architecture, with TraCI [34]. This feature is of interest because it enables external applications to control vehicle parameters on run time, such as platoon leaders. This allows to research and develop new methods for vehicle cooperation and coordination, while the SUMO acts as the simulation engine for the platoon control. SUMO is a traffic simulator that uses the Gipps-model extension and the IDM, which mimics human behavior. However, we needed to change the car following model, to enable vehicles to create and maintain platoons with constant spacing, independently of their velocities. As such, the control of the vehicles was implemented in two major steps: 1) the platoon leaders' parameters are controlled externally with the TraCI package, which allows their change in run time, enabling platoons control through their leaders; 2) the remain vehicles are controlled by the SUMO itself, through a new module that implements the longitudinal control model of the vehicles, described in Section III. The simulation platform works at the subsecond level.

\section{SIMULATION SCENARIO}

The microscopic simulation scenario consisted of a lane with approximately 5 kilometers long. The initial setup consisted of the launching of eight vehicles, with a length of 3 meters each, a few seconds apart. The first one becomes the leader and the followers obey to the control model implemented for the approach of the preceding vehicles, until they are almost one meter apart. Then, the speed profile of Fig. 3 is applied to the leader's motion with external commands made through TraCI, and the spacing error between the platoon vehicles is monitored and analyzed.

Subsequent simulations were performed with several platoons simultaneously. Fig. 1 shows the initial phase of one of those simulations performed.

\section{RESULTS}

The simulation output was analyzed and the results are shown through Fig. 2 to 5 .

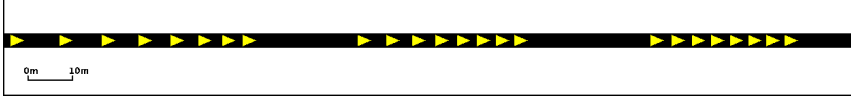

Fig. 1. Three platoons in formation. In the first platoon almost all vehicles are already in place. In the middle platoon, the vehicles at the platoon's tail are still in the approaching maneuver. The last platoon are in its initia phase of formation.

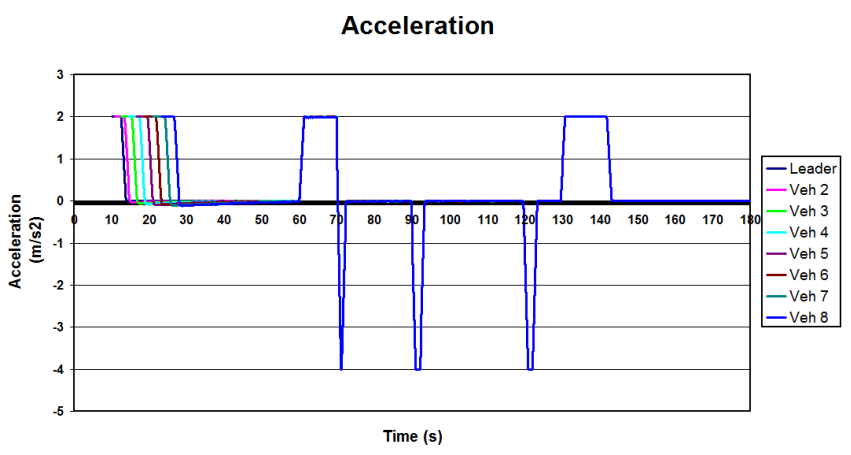

Fig. 2. Acceleration of the leader and the remaining seven vehicles of the platoon. The maximum acceleration is bounded by $-4 \leq a \leq 2 \mathrm{~m} / \mathrm{s}^{2}$.

In Fig. 2, we present the acceleration applied to the leader and the resultant acceleration patterns of the remaining vehicles, under the controller in (5), computed in the simulation platform SUMO. We limit the acceleration between $-4 m / s^{2}$ to $2 \mathrm{~m} / \mathrm{s}^{2}$. Initially, a $2 \mathrm{~m} / \mathrm{s}^{2}$ acceleration is applied to the leader until it reach a speed of $5 \mathrm{~m} / \mathrm{s}$. Since then, the leader acceleration is maintained at zero for some time. We can see that the following vehicles, which departed with some delay, adapt their acceleration patterns to approach their precedent vehicle, by maintaining the maximum allowed acceleration value for some time, braking slightly afterwards to conclude the approaching procedure.

In Fig. 3, we can see that the followers' speed becomes greater than the leader's, to enable the approaching maneuver from the initial phase of the simulation until $60 \mathrm{~s}$. The farthest each vehicle is to the leader, the greatest the amplitude of speed and braking effort.

In Fig. 4, we can observe the distance made by all vehicles and confirm that they are getting closer to each other. The spacing errors of the vehicles, with respect to the $1 \mathrm{~m}$ objective, are depicted in Fig. 5. The vehicles reach a maximum distance from their precedent of about $5.5 \mathrm{~m}$ (2nd vehicle), to $12.9 \mathrm{~m}$ (8th vehicle). However, as the vehicles approach their precedent vehicle and the leader, all errors fall bellow $1 m$ after $70 s$, and approach zero. At $60 s$, the leader's acceleration becomes $2 \mathrm{~m} / \mathrm{s}^{2}$ again, until it reaches $25 \mathrm{~m} / \mathrm{s}$ at $70 s$. Note that, even then, the spacing error between vehicles continues to decrease. After $172 s$, all vehicles present zero spacing errors.

The obtained results confirm that the system behaves as expected from the analytical analysis [11]. After the platoon formation stabilizes, the eight vehicles follow the leader with one meter apart, and the spacing errors are negligible. 
Speed

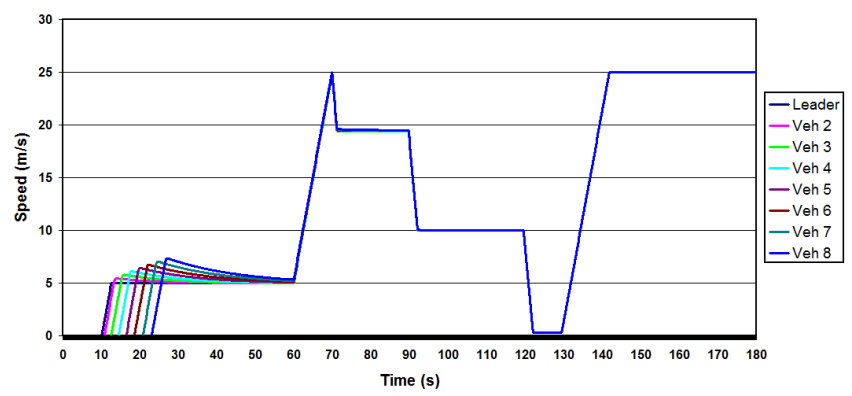

Fig. 3. Velocity of the leader and the remaining seven vehicles of the platoon. Note that, after $60 s$, all vehicles follow the leader with the same speed pattern.

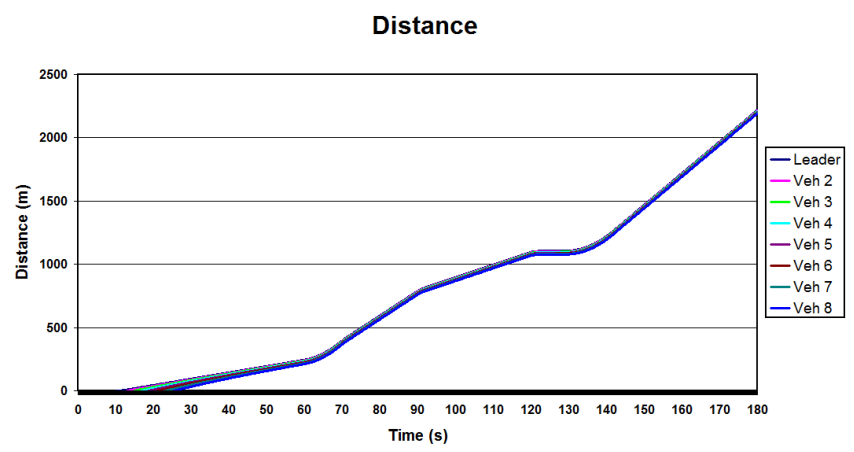

Fig. 4. Distance of the leader and the remaining seven vehicles of the platoon. Note that, after $60 \mathrm{~s}$, all vehicles follow the leader very close to each other

\section{ONGOING WORK AND CONCLUSION}

A simulation engine for platoons of autonomous vehicles was implemented in the SUMO. For that purpose, the simulator was modified to operate with a new car following model. A simulation scenario was defined and the results of the simulations confirm the correctness and robustness of the model.

To assess how the communication delays affect the platoon stability, the following communication and information update schemes are subject of study.

Communication Scheme: The communication architecture of the platoon vehicles uses time division multiple access with the token passing [26]. All vehicles transmit their data at each token cycle $t c$, in their respective time slot $t s$. For that purpose, $t c_{i}=10 \times t s_{j}, j=1, \ldots, 10$. We also consider $t c=100 \mathrm{~ms}$ and $t s=10 \mathrm{~ms}$ as appropriate reference values.

Information Updating Scheme I: The platoon leader announce the plan for the next action, but it and all remaining platoons vehicles hold their actuation until all vehicles receive its feedback. After that, all vehicles update their operation parameters simultaneously [26], in each information update cycle $u c$. With this information update scheme, we aim to compensate the effects that the communication delays might have on the platoon stability. However, with a $n$-vehicles platoon, each $u c$ should attain the condition $u c \geq n \times t c$.
Spacing error

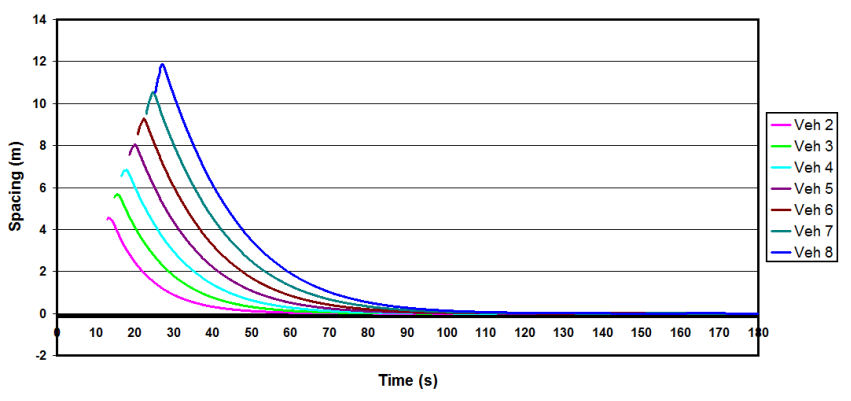

Fig. 5. Spacing error. After $70 s$, it falls bellow $1 m$ for all vehicles of the platoon.

Information Updating Scheme II: Since all vehicles in the platoon receive information from the leader at all $t c$, it is envisaged to evaluate the feasibility and effect of the variation of the parameter $C_{1}$ in (5), assigning more weight on the leader's data under some potential dangerous conditions (e.g., in the presence of a strong deceleration of the leader), and allowing a faster platoon response (e.g., with $u c=t c$ ). For this purpose, new approaches for information update are required and under research.

Simulation and analysis of the proposed communication algorithms, aiming to demonstrate, through simulation, the validity of new mobility paradigms are under development.

\section{ACKNOWLEDGMENTS}

We would like to acknowledge Roberto Machado, for his collaboration on the changes of the SUMO traffic simulator source code.

\section{REFERENCES}

[1] P. Fernandes, and U. Nunes, "Vehicle Communications: A Short Survey", in IADIS Telecommunications, Networks and Systems, Lisboa, Portugal, July 2007.

[2] M. Treiber, A. Hennecke, D. Helbing, "Congested Traffic States in Empirical Observations and Microscopic Simulations", Physical Review E 62 (2): pp. 18051824, 2000.

[3] A. Kesting, "Microscopic Modeling of Human and Automated Driving: Towards Traffic-Adaptive Cruise Control", $\mathrm{PhD}$ Thesis, Dresden; Feb, 2008.

[4] Dedicated Short Range Communications (DSRC) Home. [Online]. http://www.leearmstrong.com/DSRC/DSRCHomeset.htm

[5] C. Palazzi, M. Roccetti, and S. Ferretti, "An Intervehicular Communication Architecture for Safety and Entertainment", IEEE Transactions on Intelligent Transportation Systems, vol. 11, no. 1, pp 90-99, March, 2010.

[6] A. Kesting, M. Treiber, and D. Helbing, "Connectivity Statistics of Store-and-Forward Intervehicle Communication", IEEE Transactions on Intelligent Transportation Systems, vol. 11, no. 1, pp 172-181, March, 2010.

[7] LTE, Long Term Evolution. [Online]. Available: http://www.3gpp.org/ LTE

[8] LTE-Advanced (Long Term Evolution). [Online]. Available: http://www.3gpp.org/LTE-Advanced

[9] K.Zeng, B. Fan, Z. Ma, G. Liu, X. Shen, and W. Wang, "Multihop Cellular Networks Toward LTE-Advanced", IEEE Vehicular Tachnology Magazine, vol. 4, no. 3, pp 40-47, September, 2009.

[10] SUMO, Simulation for Urban MObility. [Online]. Available: http://sourceforge.net/apps/mediawiki/sumo

[11] R. Rajamani, Vehicle Dynamics and Control, Springer, 2006. 
[12] J. E. Anderson, "An Intelligent Transportation Network System: Rationale, Attributes, Status, Economics, Benefits, and Courses of Study for Engineers and Planners", PRT International, LLC, 2009.

[13] NS2, The Network Simulator. [Online]. Available: http://nsnam. isi.edu/nsnam/

[14] ULTra PRT, Urban Light Transport. [Online]. Available: http://www. ultraprt.com/prt/

[15] Cybercars - Cybernetic Technologies for the Car in the City. [Online]. Available: http://cybercars2.paris-rocquencourt.inria.fr/

[16] CyberMove - Cybernetic Transportaion Systems for the Cities of Tomorrow. [Online]. Available: http://ralyx.inria.fr/2005/Raweb/imara/uid76.html

[17] CyberC3 - Cybernetic technologies for Cars in the Chinese Cities. [Online]. Available: http://cyberc3.sjtu.edu.cn

[18] PATH, California Partners for Advanced Transit and Highways. [Online]. Available: http://www.path.berkeley.edu/

[19] P. Varaiya, "Smart cars on smart roads: problems of control", Transactions on Automatic Control, AC-38(2), pp. 195-207, 1993.

[20] D. Swaroop, "String Stability of Interconnected Systems: An Application to Platooning in Automated Highway Systems", Ph.D. Thesis. University of California, Berkeley, 1994.

[21] L. Alvarez, and R. Horowitz, "Safe Platooning in Automated Highway Systems", California PATH Research Report no. UCB-ITS-PRR-9746, 1997.

[22] R. Horowitz, and P. Varaiya "Control Design of an Automated Highway System", Proceedings of the IEEE: Special Issue on Hybrid Systems,, vol. 88, no. 7, pp. 913-25, July 2000.

[23] R. Rajamani, H. S. Tan, B. Law, and W. B. Zhang, "Demonstration of Integrated Lateral and Longitudinal Control for the Operation of Automated Vehicles in Platoons", IEEE Transactions on Control Systems Technology, Vol. 8, No. 4, pp. 695-708, July 2000.

[24] J. Slotine and W. Li, Vehicle Dynamics and Control, Prentice Hall, 1991.

[25] S. Mahal, "Effects on Communication Delays on String Stability in a AHS Environment", M.S. Thesis, UC Berkeley, March 2000.

[26] X. Liu, and A. Goldsmith, "Effects on Communication Delay on String Stability in Vehicle Platoons", in Proceedings of IEEE 4th International Conference of Intelligent Transportation Systems (ITSC), Oakland, USA, 2001.
[27] M. Balmer, M. Rieser, K. Meister, D. Charypar, N. Lefebvre, K Nagel, and K.W. Axhausen, "MATSim-T: Architecture and Simulation Times", in A. L. C. Bazzan and F. Klgl (eds.) Multi-Agent Systems for Traffic and Transportation Engineering, pp. 5778, Information Science Reference, Hershey, 2009.

[28] MATSIM, Multi-Agent Transport Simulation Toolkit. [Online]. Available: http://www.matsim.org/

[29] D. Krajzewicz, G. Hertkorn, C. Rössel, and P. Wagner, "SUMO (Simulation of Urban MObility); An Open-source Traffic Simulation", in Proceedings of the 4th Middle East Symposium on Simulation and Modelling (MESM2002), SCS European Publishing House, pp. 183187, Sharjah, United Arab Emirates, Sept. 2002.

[30] D. Krajzewicz, M. Bonert, and Peter Wagner, "The Open Source Traffic Simulation Package SUMO”, RoboCup 2006 Infrastructure Simulation Competition, RoboCup 2006, Germany, Bremen, 2006.

[31] Stefan Krauß. Microscopic Modeling of Traffic Flow: Investigation of Collision Free Vehicle Dynamics". PhD Thesis, Universität zu Köln, April 1998.

[32] T. Kosh, S. Eichler, B. Ostermeier, and C. Schroth, "Simulation of Car-to-Car Messaging: Analysing the Impact on Road Traffic", in 13th IEEE Symposium on Modeling, Analysis, and Simulation of Computer and Telecommunication Systems, pp. 507-510, 2005.

[33] M. Piorkowski, M. Raya, A. Lugo, P. Papadimitratos, M. Grossglauser, and J.-P. Hubaux, "TraNS: Realistic Joint Traffic and Network Simulator for VANETs", ACM SIGMOBILE Mobile Computing and Communications Review, pp. 31-33, 2008

[34] A. Wegener, M. Piorkowski, M. Raya, H. Hellbrck, S. Fischer, and J.-P. Hubaux, "TraCI: An Interface for Coupling Road Traffic and Network Simulators", in 11th Communications and Networking Simulation Symposium (CNS), 2008.

[35] Hermes Network Simulator. [Online]. Available: http://students.ceid. upatras.gr/xithalis/simulation_en.html

[36] innovative Transportation Simulator (iTS). [Online]. Available: http://www.maitint.org/

[37] D. Krajzewicz, G. Hertkorn, C. Rössel, and P. Wagner, “An Example of Microscopic Car Models Validation using the open source Traffic

Simulation SUMO", in Proceedings of Simulation in Industry, 14th European Simulation Symposium, 2002, SCS European Publishing House, pp. 318-322, Dresden, Germany, Oct. 2002. 